\title{
Characterization of the transcriptional response of Candida parapsilosis to the antifungal peptide MAF-1A
}

\author{
Rong Cheng ${ }^{1}$, Wei Li ${ }^{2}$, Klarke M. Sample ${ }^{3,4}$, Qiang Xu ${ }^{3,4}$, Lin Liu ${ }^{4,5}$, Fuxun Yu ${ }^{3,4}$, Yingjie Nie ${ }^{3,4}$, Xiangyan Zhang \\ Corresp., 4, 5 , Zhenhua Luo ${ }^{\text {Corresp. 3, } 4}$ \\ ${ }^{1}$ Guizhou University School of Medicine, Guiyang, China \\ 2 Department of Cadiovascular Medicine, Affiliated Hospital of Guizhou Medical University, Guiyang, China \\ 3 Department of Central Lab, Guizhou Provincial People's Hospital, Guiyang, China \\ 4 NHC Key Laboratory of Pulmonary Immune-related Diseases, Guizhou Provincial People's Hospital, Guiyang, China \\ 5 Department of Respiratory and Critical Care Medicine, Guizhou Provincial People's Hospital, Guiyang, China \\ Corresponding Authors: Xiangyan Zhang, Zhenhua Luo \\ Email address: zxiangyan12@sina.com, luo8300@sina.com
}

Candida parapsilosis is a major fungal pathogen that leads to sepsis. New and more effective antifungal agents are required due to the emergence of resistant fungal strains. MAF-1A is a cationic antifungal peptide isolated from Musca domestica that is effective against a variety of Candida species. However, the mechanism(s) of its antifungal activity remains undefined. Here, we used RNA-seq to identify differentially expressed genes (DEGs) in Candida parapsilosis following MAF-1A exposure. The early (6 h) response included 1122 upregulated and 1065 downregulated genes. Late $(18 \mathrm{~h})$ responses were associated with the increased expression of 101 genes and the decreased expression of 151 genes. Upon MAF-1A treatment for 18 h, 42 genes were upregulated and 25 genes were downregulated. KEGG enrichment showed that the DEGs in response to MAF-1A were mainly involved in amino acid synthesis and metabolism, oxidative phosphorylation, sterol synthesis, and apoptosis. These results indicate that MAF-1A exerts antifungal activity through interference with Candida parapsilosis cell membrane integrity and organelle function. This provides new insight into the interaction between Candida parapsilosis and this antimicrobial peptide and serves as a reference for future Candida parapsilosis therapies. 


\section{Characterization of the Transcriptional Response of Candida}

2 parapsilosis to the Antifungal Peptide MAF-1A

3 Rong Cheng', Wei Li², Klarke M. Sample, ${ }^{3,4}$, Qiang Xü ${ }^{3,4}$, Lin Liu ${ }^{4,5}$, Fuxun Yu ${ }^{3,4}$, Yingjie

$4 \mathrm{Nie}^{3,4}$, Xiangyan Zhang ${ }^{4,}$ * Zhenhua $\mathrm{Luo}^{3,4}$ *

$5{ }^{1}$ Guizhou University School of Medicine, Guiyang, China.

$6{ }^{2}$ Department of Cadiovascular Medicine, Affiliated Hospital of Guizhou Medical University,

7 Guiyang, China.

$8{ }^{3}$ Department of Central Lab, Guizhou Provincial People's Hospital; Guiyang, China.

$9 \quad{ }^{4}$ NHC Key Laboratory of Pulmonary Immune-related Diseases, Guizhou Provincial People's

10 Hospital, Guiyang, China.

${ }^{5}$ Department of Respiratory and Critical Care Medicine, Guizhou Provincial People's Hospital, Guiyang, China.

*Corresponding Authors: Xiangyan Zhang, Zhenhua Luo

Email address: zxiangyan12@sina.com, luo8300@sina.com

Abstract: Candida parapsilosis is a major fungal pathogen that leads to sepsis. New and more effective antifungal agents are required due to the emergence of resistant fungal strains. MAF-1A is a cationic antifungal peptide isolated from Musca domestica that is effective against a variety of Candida species. However, the mechanism(s) of its antifungal activity remains undefined. Here, we used RNA-seq to identify differentially expressed genes (DEGs) in Candida parapsilosis following MAF-1A exposure. The early $(6 \mathrm{~h})$ response included 1122 upregulated and 1065 downregulated genes. Late $(18 \mathrm{~h})$ responses were associated with the increased expression of 101 genes and the decreased expression of 151 genes. Upon MAF-1A treatment for $18 \mathrm{~h}, 42$ genes were upregulated and 25 genes were downregulated. KEGG enrichment showed that the DEGs in response to MAF-1A were mainly involved in amino acid synthesis and metabolism, oxidative phosphorylation, sterol synthesis, and apoptosis. These results indicate that MAF-1A exerts antifungal activity through interference with Candida parapsilosis cell membrane integrity and organelle function. This provides new insight into the interaction between Candida parapsilosis and this antimicrobial peptide and serves as a reference for future Candida parapsilosis therapies.

\section{Introduction}

Immunosuppressed patients are at a high risk of hospital-acquired fungal infections. Candida albicans (C. albicans) is the most common pathogen of Candida species, its dominance has decreased as the incidence of non-albicans Candida (NAC) species have increased (Vieira de 
Melo et al. 2019). Over the last two decades, epidemiological studies of fungal pathogens have shown that NAC has surpassed C. albicans as the most prevalent cause of invasive Candida (Sular et al. 2018). New anti-NAC treatment regimens are therefore urgently required.

Amongst NAC infections, Candida parapsilosis (C. parapsilosis) is particularly problematic due to its propensity to form biofilms on central venous catheters and other medically implanted devices (Fais et al. 2017; Vieira de Melo et al. 2019). Additionally, patients in the intensive care unit (ICU) who have undergone total parenteral nutrition are highly susceptible to $\mathrm{C}$. parapsilosis infection, including undernourished children and neonates of low-birth-weights. Recent epidemiological studies have shown that C. parapsilosis is the second most commonly isolated species following only $\mathrm{C}$. albicans in southern Europe, some regions of Asia, and Latin America (Toth et al. 2019). When immunosuppressed patients are exposed to C. parapsilosis, the rate of infection is high. The biological characteristics of infection, include toxicity, immune regulation, and drug resistance are in contrast with those of C. albicans (Toth et al. 2019). These interspecies specificities affect recognition by the host, clearance, and antifungal drug efficacy.

Candida pathogens have developed varying degrees of drug resistance, with some representing a serious threat to human health (Robbins et al. 2017). The currently available antifungal agents inhibit cell wall synthesis (echinocandins), destroy cell membrane components (azoles), or bind to ergosterol and perforate the cell membrane (amphotericin B). With the widespread use of antifungal drugs, the presence of drug resistance-related genes has increased. Antimicrobial peptides (AMPs) form a key arm of the innate immune response of a variety of organisms including plants, insects, and humans (Moravej et al. 2018). It is uncommon for microbial infections to be resistant to AMPs which are an emerging source of novel antifungal drugs (Ghosh et al. 2019; Nuti et al. 2017; Patocka et al. 2018), making these molecules a potential alternative to fungemia therapies.

AMPs can exhibit both cationic and amphiphilic properties. Cationic AMPs are amphipathic permitting their interaction with negatively charged cell membranes, leading to cell membrane disruption and cell death (Kobbi et al. 2018). AMPs are diverse with respect to length (20-100 amino acids), sequence and structure, and are produced by almost all organisms. Filamentous fungi produce a wide spectrum of AMPs that serve as defense and/or host signaling molecules. Penicillium chrysogenum secretes PAF and PAFB that possess complex tertiary structures and activity centers. PAF and PAFB are produced as 92 amino acid preproproteins that are active against a variety of pathogenic fungi, bacteria, and viruses (Huber et al. 2020). Insects are extremely resistant to microbial infections, which are an important source of AMPs. Insect AMPs are smaller (between 12 and 50 amino acids) with secondary structures formed predominantly of $\alpha$-helices and $\beta$-sheets. Whilst membrane damage is the canonical mechanism through which AMPs act, other mechanisms exist. AMPs have specific subcellular targets, including the inhibition of DNA synthesis, RNA synthesis, protein synthesis, and cell wall integrity (Guilhelmelli et al. 2013; Li et al. 2016). However, their mechanism(s) of action at the molecular level remain unclear. The Musca domestica antifungal peptide-1 (MAF-1) is a novel 
cationic AMP isolated from the instar larvae of houseflies (Fu et al. 2009). We previously cloned the full-length MAF-1 gene and derived 26 amino acid MAF-1A peptides from the MAF-1 structural domain. Despite the established antifungal effects of MAF-1A, the molecular mechanism(s) governing its activity remain largely undefined (Zhou et al. 2016).

In recent years, the development of high throughput sequencing technologies has facilitated research on both antimicrobial drug function and drug-resistance. For example, HAC1 (CPAR2_103720) is a key mediator of endoplasmic reticulum stress in C. parapsilosis identified through RNA-seq (Iracane et al. 2018). In our previous studies, we showed that MAF-1A inhibits C. albicans through its effects on the cell wall, plasma membrane, protein synthesis, and energy metabolism (Wang et al. 2017). However, the mechanism(s) through which C. albicans responds to MAF-1A were not fully defined. Here, we have expanded our knowledge on how MAF-1A acts on C. parapsilosis and investigated differences in the responses of C. albicans and C. parapsilosis to MAF-1A treatment. RNA-seq was used to investigate changes in gene expression at early $(6 \mathrm{~h})$ and late $(18 \mathrm{~h})$ time points, according to time-kill curves of $\mathrm{C}$. parapsilosis growth.

\section{Materials and methods}

2.1. Strains and growth conditions

Transcriptional profiling was performed on the C. parapsilosis reference strain ATCC22019. The strain was preserved in goat blood and stored at $-80^{\circ} \mathrm{C}$. C. parapsilosis was streaked on Sabouraud Dextrose Agar (SDA) plates (Sangon, Shanghai, China) at $35^{\circ} \mathrm{C}$ as described by Lis et al (Lis et al. 2010). MAF-1A treatments were performed in Sabouraud Dextrose Broth (SDB) (Sangon, Shanghai, China).

\subsection{Peptide synthesis}

MAF-1A was synthesized by Sangon Biotech (Shanghai, Shanghai, China) as a linear peptide of 26 amino acids: KKFKETADKLIESAKQQLESLAKEMK. Analytical highperformance liquid chromatography (HPLC) was used to confirm purity $\geqslant 95 \%$. The peptide was dissolved in sterile ultrapure water at $5 \mathrm{mg} / \mathrm{mL}$ and stored at $-20^{\circ} \mathrm{C}$.

\subsection{Minimum inhibitory concentration (MIC) and time-kill curves}

Antifungal assays were performed as per the requirements of the Clinical and Laboratory Standards Institute (CLSI) M27-A3. Briefly, cultures were grown for $24 \mathrm{~h}$ at $35{ }^{\circ} \mathrm{C}$ and resuspended in SDB. Concentrations were adjusted to approximately $0.5 \times 10^{3}-2.5 \times 10^{3}$ $\mathrm{CFU} / \mathrm{mL}$ and $100 \mu \mathrm{l}$ of the suspension was added to each well of 96-well polypropylene microplates (NEST, Wuxi, China). MAF-1A was added at concentration ranging from 0.1 $\mathrm{mg} / \mathrm{mL}$ to $1.2 \mathrm{mg} / \mathrm{mL}$. All experiments were performed in triplicates. After incubation at $35^{\circ} \mathrm{C}$ for $24 \mathrm{~h}$, absorbances were measured at $492 \mathrm{~nm}$ on a Microplate Reader (BioTek Synergy H1, 
108

109

110

111

112

113

114

115

116

117

118

119

120

121

122

123

124

125

126

127

128

129

130

131

132

133

134

135

136

137

138

139

140

141

142

Vermont, USA). MIC was defined as the lowest drug concentration showing $80 \%$ growth inhibition compared to the drug-free controls. The following formulas were used (Li et al. 2008):

(1) Percentage Fungal Growth $=($ Treatment Well A Value - Control Well A Value $) /$ (Growth in Control Well A Value - Control Well A Value) $\times 100 \%$

(2) Percentage Inhibition of Fungal Growth $=1$ - Percentage Fungal Growth

Time-kill curves were performed according to the literature (Li et al. 2008; Sun et al. 2008). C. parapsilosis suspensions were mixed with MAF-1A (MIC) in triplicate and cultured at $35^{\circ} \mathrm{C}$. Aliquots of $100 \mu \mathrm{l}$ were removed from each test solution at pre-determined time points $(0,2,4,6$, $8,10,12,14,16,18,20,22$, and $24 \mathrm{~h})$. Dilutions were produced $(1: 100)$ and streaked in triplicate onto SDA agar plates for colony counts after incubation at $35^{\circ} \mathrm{C}$ for $24 \mathrm{~h}$. Sterile ultrapure water was used as a control.

\subsection{Transcriptome sequencing}

C. parapsilosis was inoculated into SDB medium (Sangon, Shanghai, China) at $35^{\circ} \mathrm{C}$ for 24 h. C. parapsilosis was treated with MAF-1A at MIC for $6 \mathrm{~h}$ (CPAS) and $18 \mathrm{~h}$ (CPBS), before RNA extraction. Untreated cultures served as controls (6 h, CPAC; $18 \mathrm{~h}, \mathrm{CPBC})$. Total RNA was extracted using RNAiso Plus (Takara, Dalian, China) according to the manufacturer's instructions. RNA concentration and quality were determined on a NanoDrop 2000 (Thermo Fisher Scientific, Wilmington, DE, USA) and Agilent 2100 bioanalyzer (Agilent Technologies, CA, USA). Libraries were prepared using NEBNext@ UltraTM RNA Library Prep Kit (NEB, USA) as per the manufacturer's recommendations. Purified libraries were quantified on an Agilent 2100 bioanalyzer Effective concentrations were determined through qRT-PCR analysis. Libraries were prepared and sequenced using a Novoseq sequencer (Illumina, USA) to produce 150 bp paired-end reads.

\subsection{Differential expression analysis}

Raw reads were filtered to obtain high-quality clean reads for subsequent analysis. All reads were mapped to the reference genome of C. parapsilosis (assembly ASM18276v2) from the National Center for Biotechnology Information (NCBI) using HISAT2 v2.0.5 (Kim et al. 2015). Differential expression analysis between the conditions was assessed using the Bioconductor software package DESeq2 in R 1.16.1 (Love et al. 2014). Relative gene expression was assessed using FPKM (Fragments Per Kilobase of transcript sequence per Millions of base pairs sequenced) and compared using $\log 2$ FC. P-values were adjusted to generate false discovery rates (padj) as described by Benjamini-Yekutieli, assigning the significance threshold for DEGs as padj < 0.05 (Benjamini et al. 2001; Mortazavi et al. 2008).

2.6. Enrichment and interaction network analysis of the differentially expressed genes

To further understand the functions of the DEGs, Gene Ontology (GO) enrichment was 
performed using the Bioconductor software clusterProfiler 3.4.4 in R package (Yu et al. 2012). Statistical enrichment of the DEGs was also performed in the Kyoto Encyclopedia of Genes and Genomes (KEGG) for pathway enrichment (Kanehisa et al. 2019; Ogata et al. 1999). PPI analysis of the DEGs was performed based on the STRING database to define key proteinprotein interactions ( $\mathrm{Yu}$ et al. 2012). The network was constructed using Cytoscape 3.6.1 (Shannon et al. 2003).

\subsection{Validation of RNA-seq by quantitative RT-PCR (qRT-PCR)}

To confirm the RNA-seq data, 20 DEGs (10 with increased expression and 10 with decreased expression) were selected for qRT-PCR validations. Reactions were performed using SYBR Premix Ex Taq TM Kit (Takara) according to the manufacturer's protocol. Reaction conditions were as follows: 40 cycles of $95^{\circ} \mathrm{C}$ for 30 seconds; $95^{\circ} \mathrm{C}$ for 5 seconds; and $60^{\circ} \mathrm{C}$ for 30 seconds. PCRs were performed on a BIO-RAD CFX-Connect Real-Time System. Relative gene expression was determined using the $2^{-\Delta \Delta \mathrm{Ct}}$ method normalized to $18 \mathrm{~S}$ rRNA (Livak \& Schmittgen 2001). Significant differences were determined using a t-test with a threshold of $p<$ 0.05. Primers are listed in Supplementary Table S1. Primer efficiency and melting curves are listed in Supplementary Table S2 and Figure S1-S5.

\section{Results}

\subsection{MIC assays and time-kill curves}

The MIC of MAF-1A against C. parapsilosis was determined as $0.6 \mathrm{mg} / \mathrm{mL}$. Time-kill curves of MAF-1A at MIC showed a gradual antifungal effect during the first $8 \mathrm{~h}$ of C. parapsilosis culture (Figure 1). After $8 \mathrm{~h}$, cell numbers increased but remained lower than those of the control group.

3.2. Transcriptional stress responses and enrichment analysis of MAF-1A treated C. parapsilosis

RNA-seq analysis in C. parapsilosis treated with MAF-1A for 6 and $18 \mathrm{~h}$ showed 5747 DEGs. Sequence reads were deposited in the NCBI Sequence Read Archive (SRA) under accession number PRJNA638006. A total of 2439 DEGs were detected. Out of these genes, 2187 were identified at $6 \mathrm{~h}$, representing 38.05\% of the total detectable genes. A total of 252 genes were differentially expressed after $18 \mathrm{~h}$ and accounted for $4.38 \%$ of the total expressed genes. After 6 and $18 \mathrm{~h}$ of MAF-1A treatment, 67 DEGs with opposite trends were observed (reversed genes 1, RG1 and reversed genes 2, RG2). In total, 56 DEGs were upregulated, whilst downregulated genes remained unchanged (1 unchanged genes: UG1; 2 unchanged genes: UG2 (Figure 2).

\subsubsection{DEG enrichment analysis}

Amongst the DEGs at $6 \mathrm{~h}, 1122$ showing increased expression were enriched in $85 \mathrm{KEGG}$ pathways, 20 of which were significant with padj $<0.05$. The most significant pathways with increased expression in C. parapsilosis following MAF-1A treatment were: oxidative 
179

180

181

182

183

184

185

186

187

188

189

190

191

192

193

194

195

196

197

198

199

200

201

202

203

204

205

206

207

208

209

210

211

212

213

214

215

phosphorylation, peroxisome, citrate cycle (TCA cycle), carbon metabolism, cell cycle-yeast, MAPK signaling-yeast, meiosis-yeast, fatty acid degradation, and autophagy (Table 1). Genes of decreased expression were enriched in steroid biosynthesis, biosynthesis of amino acids, cysteine and methionine metabolism, biosynthesis of antibiotics, ribosome, RNA polymerase, biosynthesis of secondary metabolites, RNA transport, ribosome biogenesis in eukaryotes, lysine biosynthesis, 2-oxocarboxylic acid metabolism, pyrimidine metabolism, glycine, serine and threonine metabolism and purine metabolism (Table 2). At $18 \mathrm{~h}, 101$ genes were upregulated and enriched in 24 KEGG pathways, two of which were significant (Table 3). A total of 151 genes were downregulated and significantly enriched in carbon metabolism, biosynthesis of antibiotics, oxidative phosphorylation, the biosynthesis of secondary metabolites, and the biosynthesis of amino acids (Table 4).

\subsubsection{RG1, RG2, UG1, and UG2 gene enrichment analysis}

The 42 genes in RG1 were enriched in 17 KEGG pathways, of which oxidative phosphorylation was most significant. Additionally, 25 genes in RG2 were enriched in 13 KEGG pathways, of which arginine biosynthesis, the biosynthesis of antibiotics, the biosynthesis of amino acids, and the biosynthesis of secondary metabolites were enriched. A total of 24 genes in UG1 and 32 genes in UG2 were enriched in 8 and 20 KEGG pathways, respectively. In UG1, the genes were enriched in butanoate metabolism, propionate metabolism, beta-alanine metabolism, valine, leucine, and isoleucine degradation. No pathways were significantly enriched in UG2 at a padj $<0.05$ (Table 5).

Genes in RG1, RG2, UG1, and UG2 were enriched in 535 GO terms, a total of 9 of which were significant (Table 6). Genes in RG1 were involved in energy production and redox processes. Genes in RG2 were associated with the anabolic processes of various organic acids. Genes in UG2 were involved in oxidation-reduction processes.

\subsection{Verification of differentially expressed genes}

A total of 20 genes were selected, including 10 with increased expression and 10 with decreased expression. Genes were evenly selected from $6 \mathrm{~h}$ and $18 \mathrm{~h}$ time points to validate the RNA-seq data by qRT-PCR. The results indicated the expression levels have a consistent change for both RNASeq and qRT-PCR. Hence, the qRT-PCR results confirmed the reliability of our RNA-Seq data (Figure 3).

\subsection{Protein-protein interaction (PPI) network analysis}

We constructed a PPI network based on the STRING database of the DEGs after $6 \mathrm{~h}$ of treatment. The PPI network contained 624 nodes and 6264 edges, with a degree filter of $\geq 10$ (Figure 4). The connectivity degree (dg) of multiple nodes in the PPI network were high, including: CpUbi1 ( $\mathrm{dg}=146)$, CpGlt1 ( $\mathrm{dg}=82), \mathrm{CpCdc} 28$ ( $\mathrm{dg}=54)$, CpCys4 (dg = 50), CpCyt1 ( $\mathrm{dg}=45)$, CpRpc40 (dg = 42), CpArx1 (dg = 42), CpDim1 (dg=41), CpYtm1 (dg = 41), CpRip1 $(\mathrm{dg}=41)$. Upon enrichment analyses the identified genes were associated with oxidative 
216 phosphorylation (Supplementary Figure S6).

217

218

219

220

221

222

223

224

225

226

227

228

229

230

231

232

233

234

235

236

237

238

239

240

241

242

243

244

245

246

247

248

249

250

\section{Discussion}

C. parapsilosis is one of the most prevalent fungal species in many regions. In addition to its high rates of infection, its etiology differs from that of C. albicans (Holland et al. 2014). Specific C. parapsilosis isolates are resistant to conventional antifungal drugs including echinocandins, azoles, and amphotericin B (Lotfali et al. 2016; Maria et al. 2018; Thomaz et al. 2018). Antimicrobial peptides lead to cell lysis and death through cell membrane leakage (Papo \& Shai 2003; Paterson et al. 2017; Shai 1999; Utesch et al. 2018). However, mechanistic studies of antimicrobial peptides have determined that their membrane interactions are complex. Park et al showed that buforin II prevents microorganisms entry into cells (Park et al. 1998). Lee et al found that antifungal $\beta$-peptides cause cell death by entering cells and causing nuclear and vacuole dysfunction (Lee et al. 2019). Chileveru et al showed that human alpha-defensin 5 enters the cytoplasm of Escherichia coli and interferes with cell division (Chileveru et al. 2015).

AMPs work through various mechanisms. In our previous studies, we showed that MAF-1A inhibits C. albicans through its effects on the cell wall, cell membrane, and ribosomes. (Wang et al. 2017). In this study, we found that MAF-1A alters gene expression in several important biological pathways in $\mathrm{C}$. parapsilosis, including oxidation-reduction processes and alternative energy sources. We further compared the response of C. albicans and C. parapsilosis to MAF-1A, most DEGs have the same expression trend (upregulated / downregulated), and identified changes in both stress and energy metabolism pathways (carbon metabolism, cell cycle, peroxisome, carbon metabolism, fatty acid degradation) (Supplementary Table S3, S4). We hypothesized that the antifungal peptide MAF-1A exerts antifungal effect and disrupts energy metabolism by affecting oxidation-reduction processes, due to its effects on the mitochondria. Whilst antimicrobial peptides have multiple modes of action, these remain undetermined for MAF-1A. Our findings suggest that intracellular targets may be the key sites of MAF-1A activity, with enrichment analysis of the DEGs suggesting that MAF-1A exerts antimicrobial activity through a variety of mechanisms.

\section{Membrane destruction}

Genes with decreased expression after $6 \mathrm{~h}$ were significantly enriched in steroid biosynthesis (Figure 5) including CpERG1, CpERG3, CpERG6, CpERG7, CpERG9, CpERG11, CpERG25, CpERG26, CpERG27, CpERG2, CpERG4, CpERG5, CpERG24, and CpSPBC16A3.12c (Kanehisa et al. 2019; Ogata et al. 1999). Azole agents exert antifungal activity by inhibiting the synthesis of ergosterol, a major component of fungal cell membranes (Ermakova \& Zuev 2017). The overexpression of ERG11 (encoding lanosterol 14-demethylase) is a major cause of azole resistance, often mediated by point mutations in the ERG11 gene. Members of the ERG gene 
251 family encode proteins involved in ergosterol biosynthesis, of which lanosterol 14-demethylase 252 is critical. In this study, MAF-1A decreased the expression of 14 genes related to sterol synthesis 253 including CPERG11, suggesting it interferes with ergosterol synthesis. Additionally, 10 genes showed increased expression after $6 \mathrm{~h}$ and were enriched in fatty acid degradation pathways (Figure 6) (Kanehisa et al. 2019; Ogata et al. 1999). Of these, the expression of CpPOX4 (CPAR2-807700) significantly increased, $(\log 2 \mathrm{FC}=1.503)$. We further verified the upregulation of these genes through qRT-PCR $(\log 2 \mathrm{FC}=2.834)$. CpPOX4 encodes a component of fatty acid biosynthesis, which indicates that the composition of the cell membrane was affected by MAF-1A.

\section{Mitochondrial function}

A total of 42 genes showed increased expression at $6 \mathrm{~h}$ and were enriched in oxidative phosphorylation pathways. The genes were mainly involved in respiratory chain electron transport processes in the mitochondrial inner membrane, including NHD release of $\mathrm{H}+$ and ATP synthase (Figure 7) (Kanehisa et al. 2019; Ogata et al. 1999). A total of six genes in RG1 were also enriched in this pathway (CpCOX15, Cpnuo-21, CpQCR2, CpQCR8, CpQCR7, and $C p C O R 1)$. Previous studies showed that $C O X 15$ encodes an indispensable mitochondrial protein for Saccharomyces cerevisiae cytochrome oxidase (Glerum et al. 1997). Cytochrome oxidase is a terminal enzyme in the respiratory electron transport chain that is essential for ATP synthesis. Reactive oxygen species (ROS) are produced by the oxidative phosphorylation of ATP and can disrupt the electron transport chain in mitochondria (Piippo et al. 2018). ROS production induces damage to lipids, proteins, lipids, and nucleic acids, leading to cell death. Eukaryotes prevent cell damage through oxidative stress detoxification and the prevention of ROS accumulation. In this study, GO enrichment analysis of the RG1 genes showed that 7 that were highly expressed were associated with redox processes (CpPOX9, CpCOX15, Српио-21, CpAAEL001134, CpQCR7, CpRGI1, and CpnamA). These processes help cells to remove accumulated ROS. Due to the increased expression of these genes in response to MAF-1A, it is possible that MAF-1A promotes oxidative phosphorylation, which disrupts electron transfer in the mitochondria, enhancing ROS production and subsequent cell damage

C.parapsilosis has an unusual mitochondrial genome architecture, consisting of linear DNA molecules of 30.9-Kbp, terminating with specific telomeric structures on both sides (738-Kbp long). This differs from telomeres at the ends of eukaryotic nuclear chromosomes, particularly in humans (Kovac et al. 1984). MAF-1A interferes with the expression of multiple genes related to the mitochondrial functions of C. parapsilosis, It is, therefore, feasible that MAF-1A interferes with the normal function of C. parapsilosis mitochondria.

\section{Conclusions}

In summary, MAF-1A has a complex response in C. parapsilosis. Most DEGs identified through RNA-seq analysis were related to oxidation-reduction processes and the use of alternative energy sources, Mitochondria are important target for the anti-fungal peptide MAF1A to exert anti-C. parapsilosis. RNA-seq data therefore provide future direction to study the antifungal mechanisms of MAF-1A and highlight the potential pathways that contribute to resistance. 
292 Acknowledgments: This study was supported by the Science and Technology Department of 293 Gui Zhou Province ((2019)2827, (2015)4015, (2018)5706); Doctoral Foundation of Guizhou 294 Provincial People's Hospital (GZSYBS(2015)12); Non-profit Central Research Institute Fund of 295 Chinese Academy of Medical Sciences (2019PT320003).

296

297

298

299

300

301

302

303

304

305

306

307

308

309

310

311

312

313

314

315

316

317

318

319

320

321

322

\section{References}

Benjamini Y, Drai D, Elmer G, Kafkafi N, and Golani I. 2001. Controlling the false discovery rate in behavior genetics research. Behav Brain Res 125:279-284.

Chileveru HR, Lim SA, Chairatana P, Wommack AJ, Chiang IL, and Nolan EM. 2015. Visualizing attack of Escherichia coli by the antimicrobial peptide human defensin 5 . Biochemistry 54:1767-1777. 10.1021/bi501483q

Ermakova E, and Zuev Y. 2017. Effect of ergosterol on the fungal membrane properties. Allatom and coarse-grained molecular dynamics study. Chem Phys Lipids 209:45-53. 10.1016/j.chemphyslip.2017.11.006

Fais R, Di Luca M, Rizzato C, Morici P, Bottai D, Tavanti A, and Lupetti A. 2017. The NTerminus of Human Lactoferrin Displays Anti-biofilm Activity on Candida parapsilosis in Lumen Catheters. Front Microbiol 8:2218. 10.3389/fmicb.2017.02218

Fu P, Wu J, and Guo G. 2009. Purification and molecular identification of an antifungal peptide from the hemolymph of Musca domestica (housefly). Cell Mol Immunol 6:245-251. 10.1038/cmi.2009.33

Ghosh C, Sarkar P, Issa R, and Haldar J. 2019. Alternatives to Conventional Antibiotics in the Era of Antimicrobial Resistance. Trends Microbiol 27:323-338. 10.1016/j.tim.2018.12.010

Glerum DM, Muroff I, Jin C, and Tzagoloff A. 1997. COX15 codes for a mitochondrial protein essential for the assembly of yeast cytochrome oxidase. J Biol Chem 272:19088-19094. $10.1074 /$ jbc. 272.30 .19088

Guilhelmelli F, Vilela N, Albuquerque P, Derengowski Lda S, Silva-Pereira I, and Kyaw CM. 2013. Antibiotic development challenges: the various mechanisms of action of antimicrobial peptides and of bacterial resistance. Front Microbiol 4:353. 10.3389/fmicb.2013.00353

Holland LM, Schroder MS, Turner SA, Taff H, Andes D, Grozer Z, Gacser A, Ames L, Haynes K, Higgins DG, and Butler G. 2014. Comparative phenotypic analysis of the major 
323

fungal pathogens Candida parapsilosis and Candida albicans. PLoS Pathog 10:e1004365. 10.1371/journal.ppat.1004365

Huber A, Galgóczy L, Váradi G, Holzknecht J, Kakar A, Malanovic N, Leber R, Koch J, Keller MA, Batta G, Tóth GK, and Marx F. 2020. Two small, cysteine-rich and cationic antifungal proteins from Penicillium chrysogenum: A comparative study of PAF and PAFB. Biochim Biophys Acta Biomembr:183246. 10.1016/j.bbamem.2020.183246

Iracane E, Donovan PD, Ola M, Butler G, and Holland LM. 2018. Identification of an Exceptionally Long Intron in the HAC1 Gene of Candida parapsilosis. mSphere 3. 10.1128/mSphere.00532-18

Kanehisa M, Sato Y, Furumichi M, Morishima K, and Tanabe M. 2019. New approach for understanding genome variations in KEGG. Nucleic Acids Res 47:D590-d595. 10.1093/nar/gky962

Kim D, Langmead B, and Salzberg SL. 2015. HISAT: a fast spliced aligner with low memory requirements. Nat Methods 12:357-360. 10.1038/nmeth.3317

Kobbi S, Nedjar N, Chihib N, Balti R, Chevalier M, Silvain A, Chaabouni S, Dhulster P, and Bougatef A. 2018. Synthesis and antibacterial activity of new peptides from Alfalfa $\mathrm{RuBisCO}$ protein hydrolysates and mode of action via a membrane damage mechanism against Listeria innocua. Microb Pathog 115:41-49. 10.1016/j.micpath.2017.12.009

Kovac L, Lazowska J, and Slonimski PP. 1984. A yeast with linear molecules of mitochondrial DNA. Mol Gen Genet 197:420-424.

Lee MR, Raman N, Ortiz-Bermudez P, Lynn DM, and Palecek SP. 2019. 14-Helical betaPeptides Elicit Toxicity against C. albicans by Forming Pores in the Cell Membrane and Subsequently Disrupting Intracellular Organelles. Cell Chem Biol 26:289-299.e284. 10.1016/j.chembiol.2018.11.002

Li L, Song F, Sun J, Tian X, Xia S, and Le G. 2016. Membrane damage as first and DNA as the secondary target for anti-candidal activity of antimicrobial peptide P7 derived from cellpenetrating peptide ppTG20 against Candida albicans. J Pept Sci 22:427-433. $10.1002 /$ psc. 2886

Li Y, Sun S, Guo Q, Ma L, Shi C, Su L, and Li H. 2008. In vitro interaction between azoles and cyclosporin A against clinical isolates of Candida albicans determined by the chequerboard method and time-kill curves. J Antimicrob Chemother 61:577-585. 
354

355

356

357

358

359

360

361

362

363

364

365

366

367

368

369

370

371

372

373

374

375

376

377

378

379

380

381

382

383

$10.1093 / \mathrm{jac} / \mathrm{dkm} 493$

Lis M, Liu TT, Barker KS, Rogers PD, and Bobek LA. 2010. Antimicrobial peptide MUC7 12mer activates the calcium/calcineurin pathway in Candida albicans. FEMS Yeast Res 10:579-586. 10.1111/j.1567-1364.2010.00638.x

Livak KJ, and Schmittgen TD. 2001. Analysis of relative gene expression data using real-time quantitative PCR and the 2(-Delta Delta C(T)) Method. Methods 25:402-408. 10.1006/meth.2001.1262

Lotfali E, Kordbacheh P, Mirhendi H, Zaini F, Ghajari A, Mohammadi R, Noorbakhsh F, Moazeni M, Fallahi A, and Rezaie S. 2016. Antifungal Susceptibility Analysis of Clinical Isolates of Candida parapsilosis in Iran. Iran J Public Health 45:322-328.

Love MI, Huber W, and Anders S. 2014. Moderated estimation of fold change and dispersion for RNA-seq data with DESeq2. Genome Biol 15:550. 10.1186/s13059-014-0550-8

Maria S, Barnwal G, Kumar A, Mohan K, Vinod V, Varghese A, and Biswas R. 2018. Species distribution and antifungal susceptibility among clinical isolates of Candida parapsilosis complex from India. Rev Iberoam Micol 35:147-150. 10.1016/j.riam.2018.01.004

Moravej H, Moravej Z, Yazdanparast M, Heiat M, Mirhosseini A, Moosazadeh Moghaddam M, and Mirnejad R. 2018. Antimicrobial Peptides: Features, Action, and Their Resistance Mechanisms in Bacteria. Microb Drug Resist 24:747-767. 10.1089/mdr.2017.0392

Mortazavi A, Williams BA, McCue K, Schaeffer L, and Wold B. 2008. Mapping and quantifying mammalian transcriptomes by RNA-Seq. Nat Methods 5:621-628. 10.1038/nmeth.1226

Nuti R, Goud NS, Saraswati AP, Alvala R, and Alvala M. 2017. Antimicrobial Peptides: A Promising Therapeutic Strategy in Tackling Antimicrobial Resistance. Curr Med Chem 24:4303-4314. 10.2174/0929867324666170815102441

Ogata H, Goto S, Sato K, Fujibuchi W, Bono H, and Kanehisa M. 1999. KEGG: Kyoto Encyclopedia of Genes and Genomes. Nucleic Acids Res 27:29-34. 10.1093/nar/27.1.29

Papo N, and Shai Y. 2003. Exploring peptide membrane interaction using surface plasmon resonance: differentiation between pore formation versus membrane disruption by lytic peptides. Biochemistry 42:458-466. 10.1021/bi0267846

Park CB, Kim HS, and Kim SC. 1998. Mechanism of action of the antimicrobial peptide buforin II: buforin II kills microorganisms by penetrating the cell membrane and inhibiting 
384

385

386

387

388

389

390

391

392

393

394

395

396

397

398

399

400

401

402

403

404

405

406

407

408

409

410

411

412

413

cellular functions. Biochem Biophys Res Commun 244:253-257. 10.1006/bbrc.1998.8159

Paterson DJ, Tassieri M, Reboud J, Wilson R, and Cooper JM. 2017. Lipid topology and electrostatic interactions underpin lytic activity of linear cationic antimicrobial peptides in membranes. Proc Natl Acad Sci U S A 114:E8324-e8332. 10.1073/pnas.1704489114

Patocka J, Nepovimova E, Klimova B, Wu Q, and Kuca K. 2018. Antimicrobial Peptides: Amphibian Host Defense Peptides. Curr Med Chem. $10.2174 / 0929867325666180713125314$

Piippo N, Korhonen E, Hytti M, Kinnunen K, Kaarniranta K, and Kauppinen A. 2018. Oxidative Stress is the Principal Contributor to Inflammasome Activation in Retinal Pigment Epithelium Cells with Defunct Proteasomes and Autophagy. Cell Physiol Biochem 49:359-367. 10.1159/000492886

Robbins N, Caplan T, and Cowen LE. 2017. Molecular Evolution of Antifungal Drug Resistance. Annu Rev Microbiol 71:753-775. 10.1146/annurev-micro-030117-020345

Shai Y. 1999. Mechanism of the binding, insertion and destabilization of phospholipid bilayer membranes by alpha-helical antimicrobial and cell non-selective membrane-lytic peptides. Biochim Biophys Acta 1462:55-70.

Shannon P, Markiel A, Ozier O, Baliga NS, Wang JT, Ramage D, Amin N, Schwikowski B, and Ideker T. 2003. Cytoscape: a software environment for integrated models of biomolecular interaction networks. Genome Res 13:2498-2504. 10.1101/gr.1239303

Sular FL, Szekely E, Cristea VC, and Dobreanu M. 2018. Invasive Fungal Infection in Romania: Changing Incidence and Epidemiology During Six Years of Surveillance in a Tertiary Hospital. Mycopathologia 183:967-972. 10.1007/s11046-018-0293-2

Sun S, Li Y, Guo Q, Shi C, Yu J, and Ma L. 2008. In vitro interactions between tacrolimus and azoles against Candida albicans determined by different methods. Antimicrob Agents Chemother 52:409-417. 10.1128/AAC.01070-07

Thomaz DY, de Almeida JN, Jr., Lima GME, Nunes MO, Camargo CH, Grenfell RC, Benard G, and Del Negro GMB. 2018. An Azole-Resistant Candida parapsilosis Outbreak: Clonal Persistence in the Intensive Care Unit of a Brazilian Teaching Hospital. Front Microbiol 9:2997. 10.3389/fmicb.2018.02997

Toth R, Nosek J, Mora-Montes HM, Gabaldon T, Bliss JM, Nosanchuk JD, Turner SA, Butler G, 
414

415

416

417

418

419

420

421

422

423

424

425

426

427

428

429

430

431

432

433

434

435

436

437

Vagvolgyi C, and Gacser A. 2019. Candida parapsilosis: from Genes to the Bedside. Clin Microbiol Rev 32. 10.1128/cmr.00111-18

Utesch T, de Miguel Catalina A, Schattenberg C, Paege N, Schmieder P, Krause E, Miao Y, McCammon JA, Meyer V, Jung S, and Mroginski MA. 2018. A Computational Modeling Approach Predicts Interaction of the Antifungal Protein AFP from Aspergillus giganteus with Fungal Membranes via Its gamma-Core Motif. mSphere 3. 10.1128/mSphere.0037718

Vieira de Melo AP, Zuza-Alves DL, da Silva-Rocha WP, Ferreira Canario de Souza LB, Francisco EC, Salles de Azevedo Melo A, and Maranhao Chaves G. 2019. Virulence factors of Candida spp. obtained from blood cultures of patients with candidemia attended at tertiary hospitals in Northeast Brazil. $J$ Mycol Med. 10.1016/j.mycmed.2019.02.002

Wang T, Xiu J, Zhang Y, Wu J, Ma X, Wang Y, Guo G, and Shang X. 2017. Transcriptional Responses of Candida albicans to Antimicrobial Peptide MAF-1A. Front Microbiol 8:894. 10.3389/fmicb.2017.00894

Yu G, Wang LG, Han Y, and He QY. 2012. clusterProfiler: an R package for comparing biological themes among gene clusters. Omics 16:284-287. 10.1089/omi.2011.0118

Zhou J, Kong L, Fang N, Mao B, and Ai H. 2016. Synthesis and Functional Characterization of MAF-1A Peptide Derived From the Larvae of Housefly, Musca domestica (Diptera: Muscidae). J Med Entomol 53:1467-1472. 10.1093/jme/tjw110 
Figure 1

Time-kill curves of MAF-1A under MIC for C. parapsilosis .

The mean growth of three C. parapsilosis cultures were recorded (Log10 CFU/ml) every $2 \mathrm{~h}$ for $24 \mathrm{~h}$

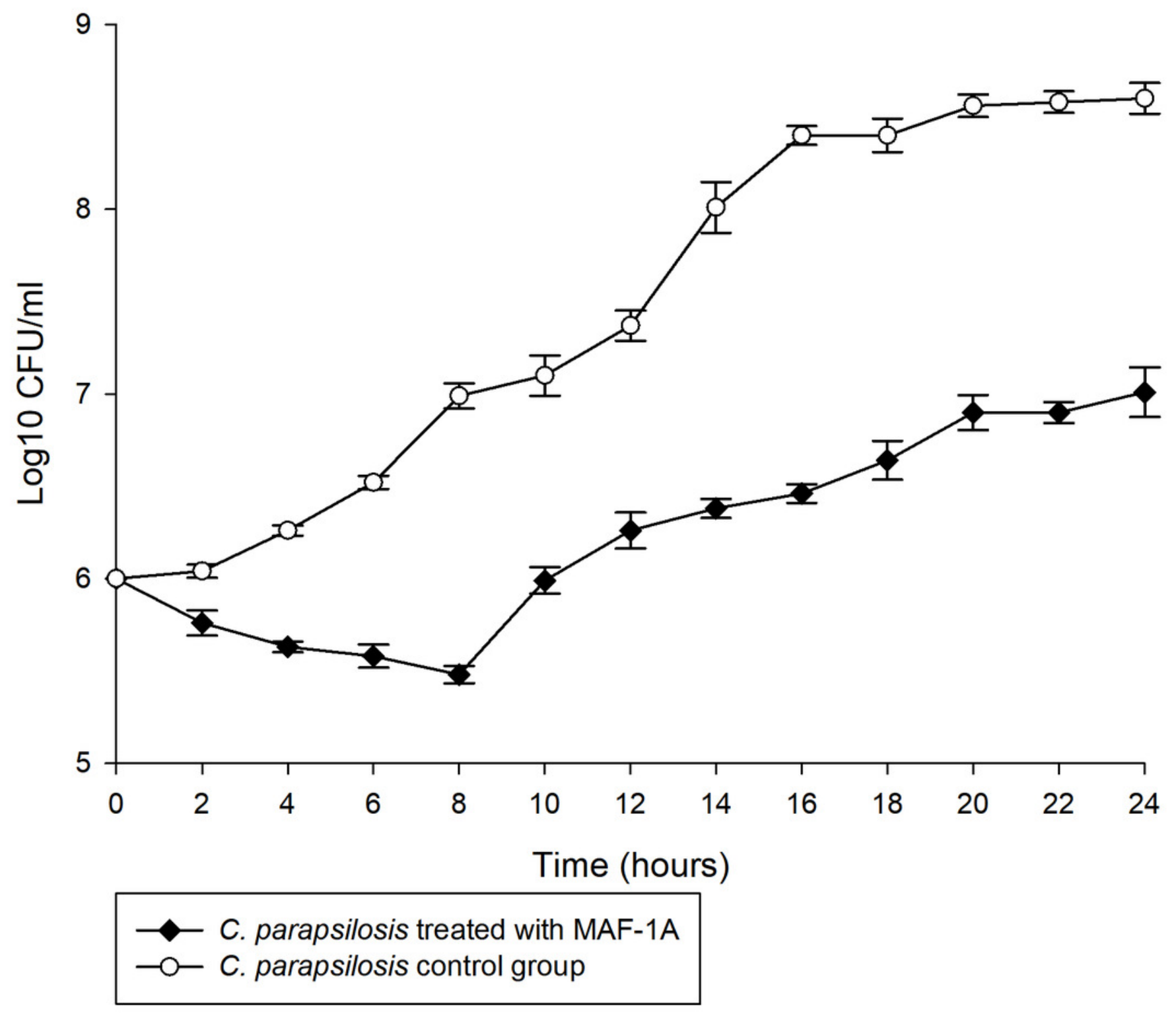




\section{Figure 2}

Gene expression changes in C. parapsilosis following MAF-1A treatment.

Volcano plots of the DEGs. A: Volcano plots depicting log2 FC (fold change) in expression after $6 \mathrm{~h}$ of treatment with MAF-1A, C. parapsilosis was treated with MAF-1A at MIC for $6 \mathrm{~h}$ (CPAS), without MAF-1A as a control (CPAC). The expression of 1122 genes significantly increased; 1065 genes were significantly downregulated (padj < 0.05). B: Volcano plot depicting the log2 FC expression after $18 \mathrm{~h}$ of treatment with MAF-1A. C. parapsilosis was treated with MAF-1A at MIC for $18 \mathrm{~h}$ (CPBS). Controls lacked MAF-1A treatment (CPBC). The expression of 101 genes significantly increased in contrast to 151 genes whose expression decreased (padj < 0.05). C: Gene expression Venn diagrams revealing two gene groups with opposite trends, labeled as RG1, RG2, UG1 and UG2; CPAS vs. CPAC_up: genes with increased expression after $6 \mathrm{~h}$; CPAS vs. CPAC down: genes with decreased expression after $6 \mathrm{~h}$; CPBS vs. CPBC_up: genes with increased expression after $18 \mathrm{~h}$; CPBS vs. CPBC down: genes with decreased expression after $18 \mathrm{~h}$. 
A

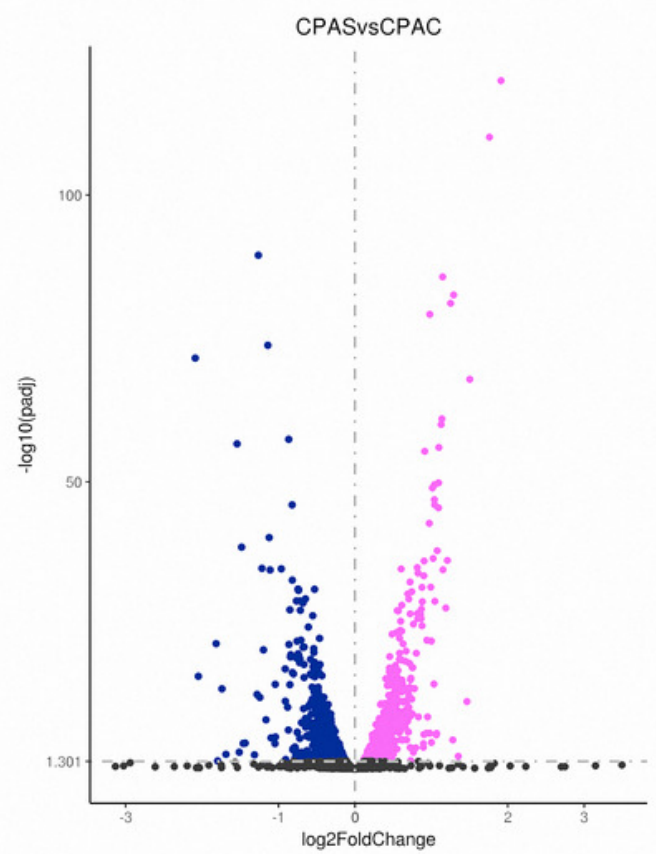

B

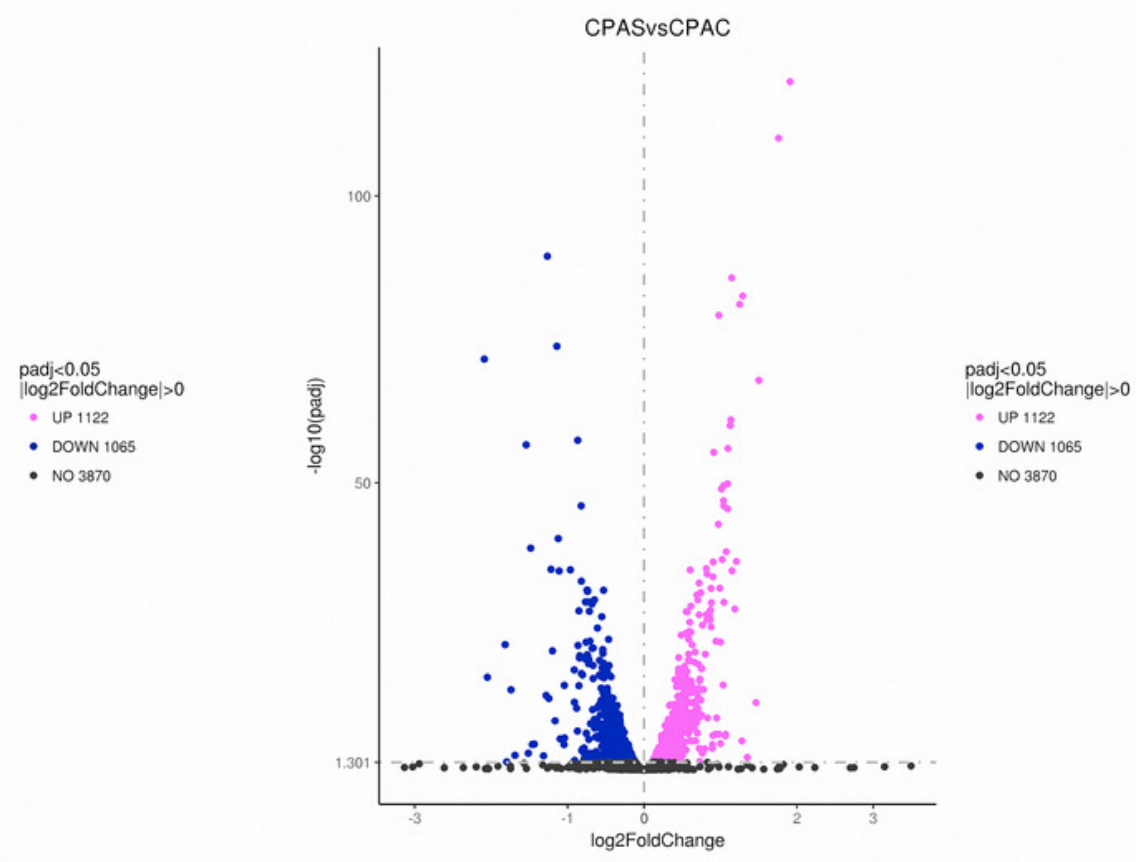

C

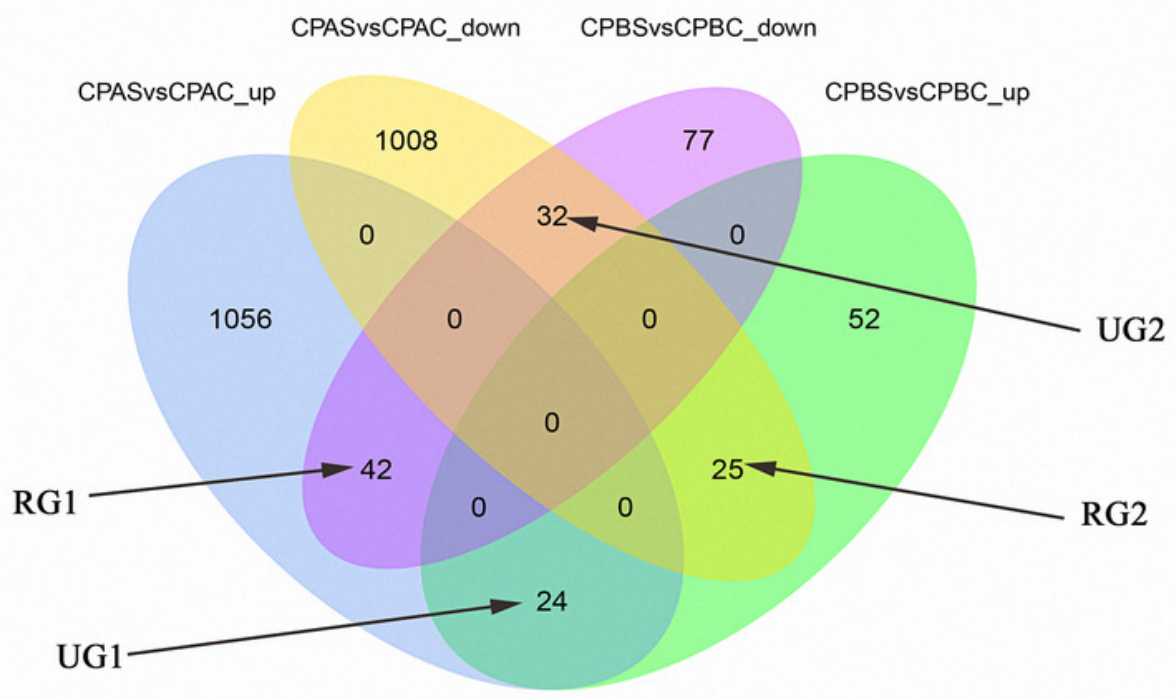


Figure 3

Validation of the RNA-seq data via qRT-PCR analysis.

A: 6 hour timepoint. B: 18 hour timepoint. $* p<0.05, * * p<0.01$, ns: not-significant. 


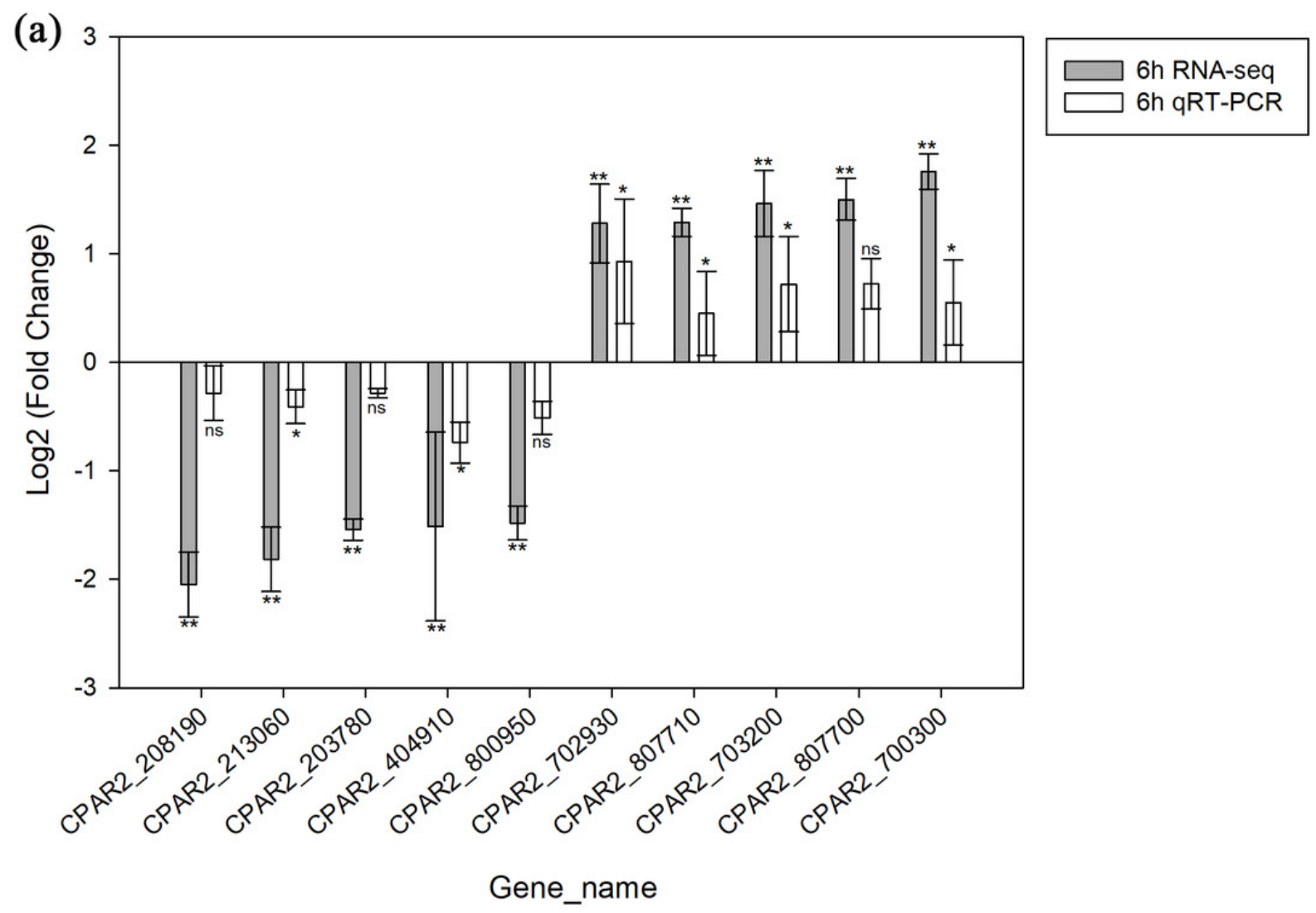

(b)

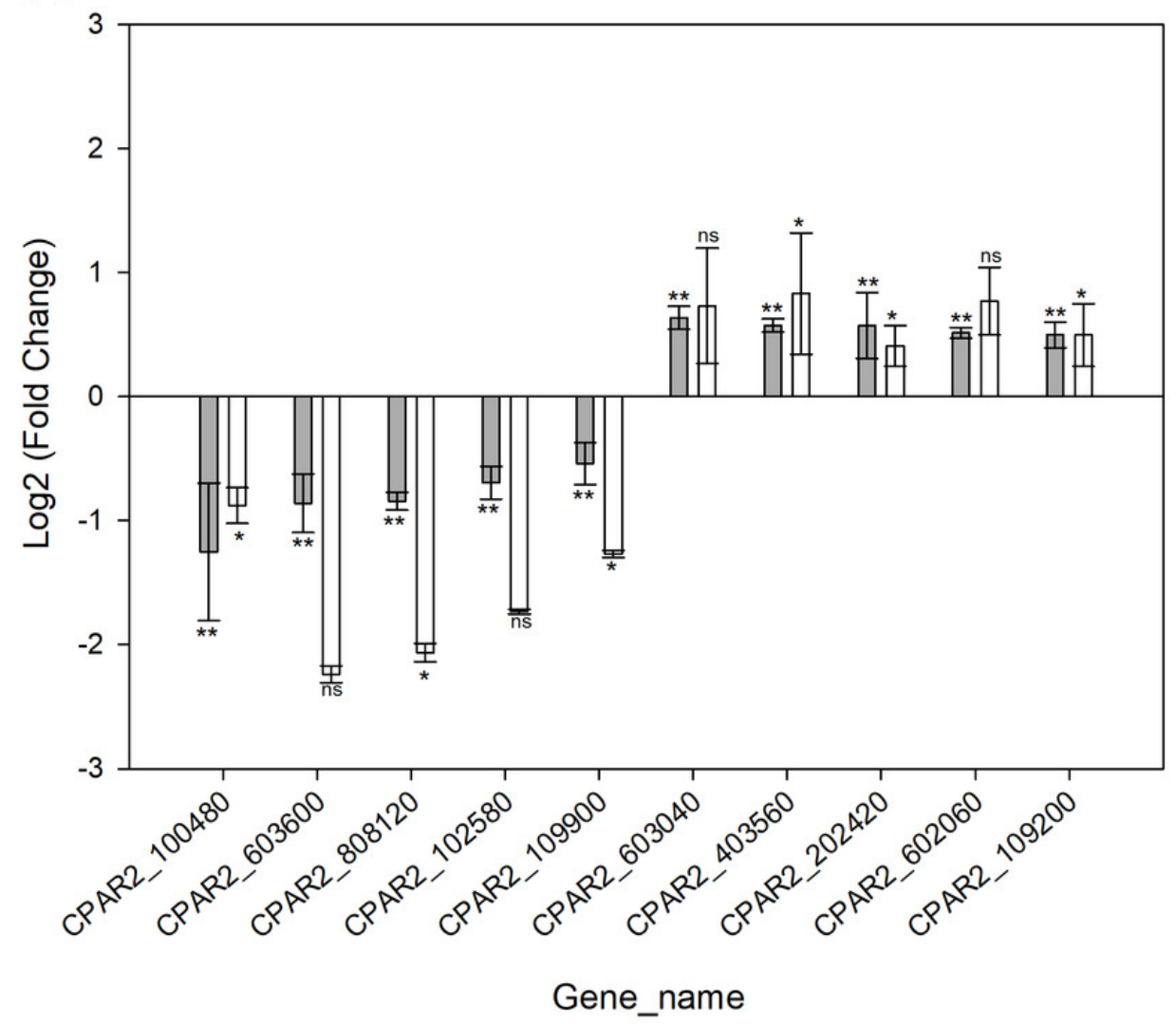




\section{Figure 4}

\section{PPI network of the DEGs following MAF-1A treatment of C. parapsilosis for $6 \mathrm{~h}$.}

Node sizes correlate with node importance; purple nodes denote genes with increased expression, and blue denote decreased expression genes.

A

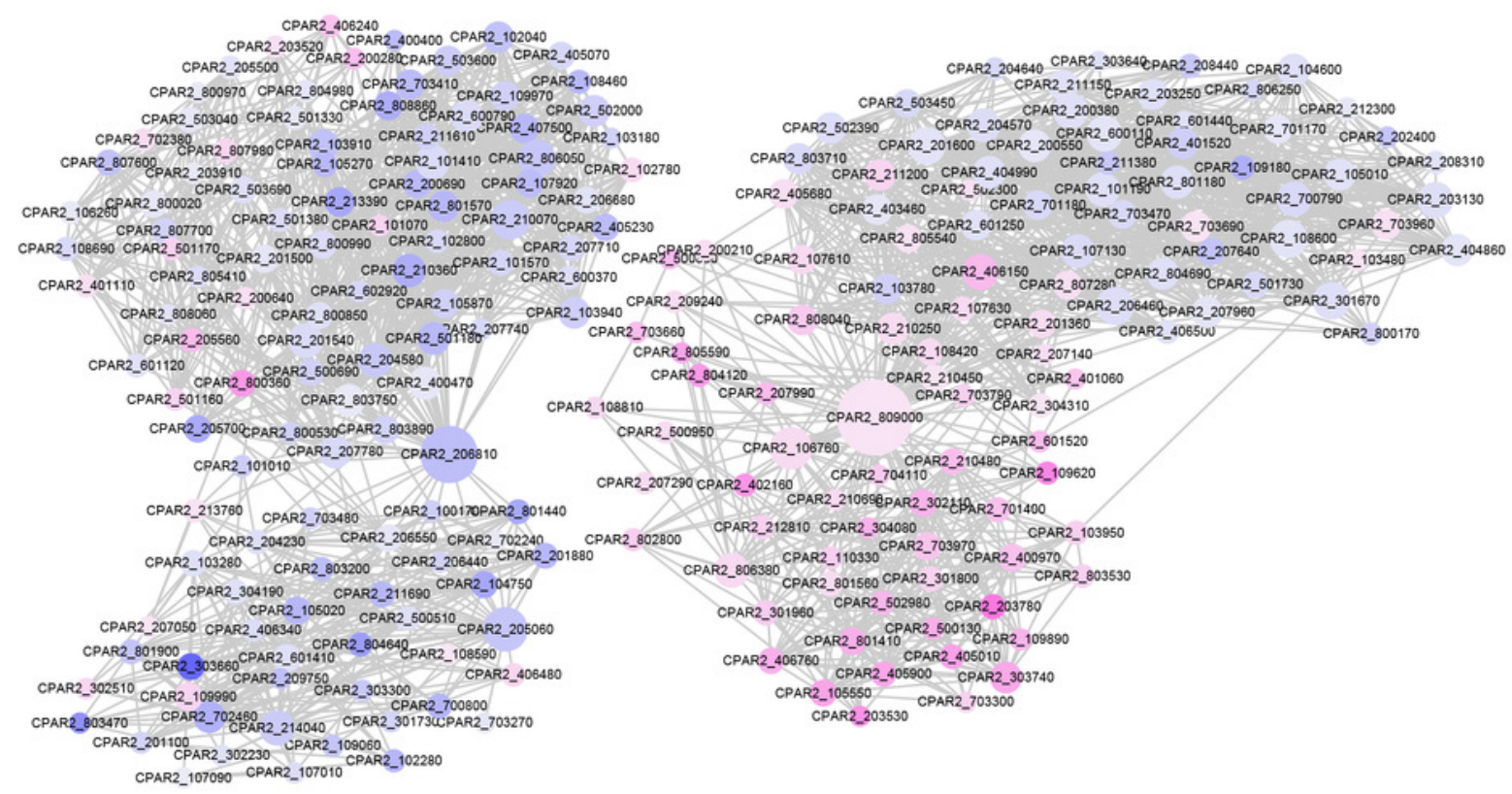




\section{Figure 5}

Significantly enriched KEGG pathways in steroid biosynthesis.

DEGs with decreased expression are shown in blue. Permission for publication granted by the Kyoto Encyclopedia of Genes and Genomes, Kyoto University, Japan. 


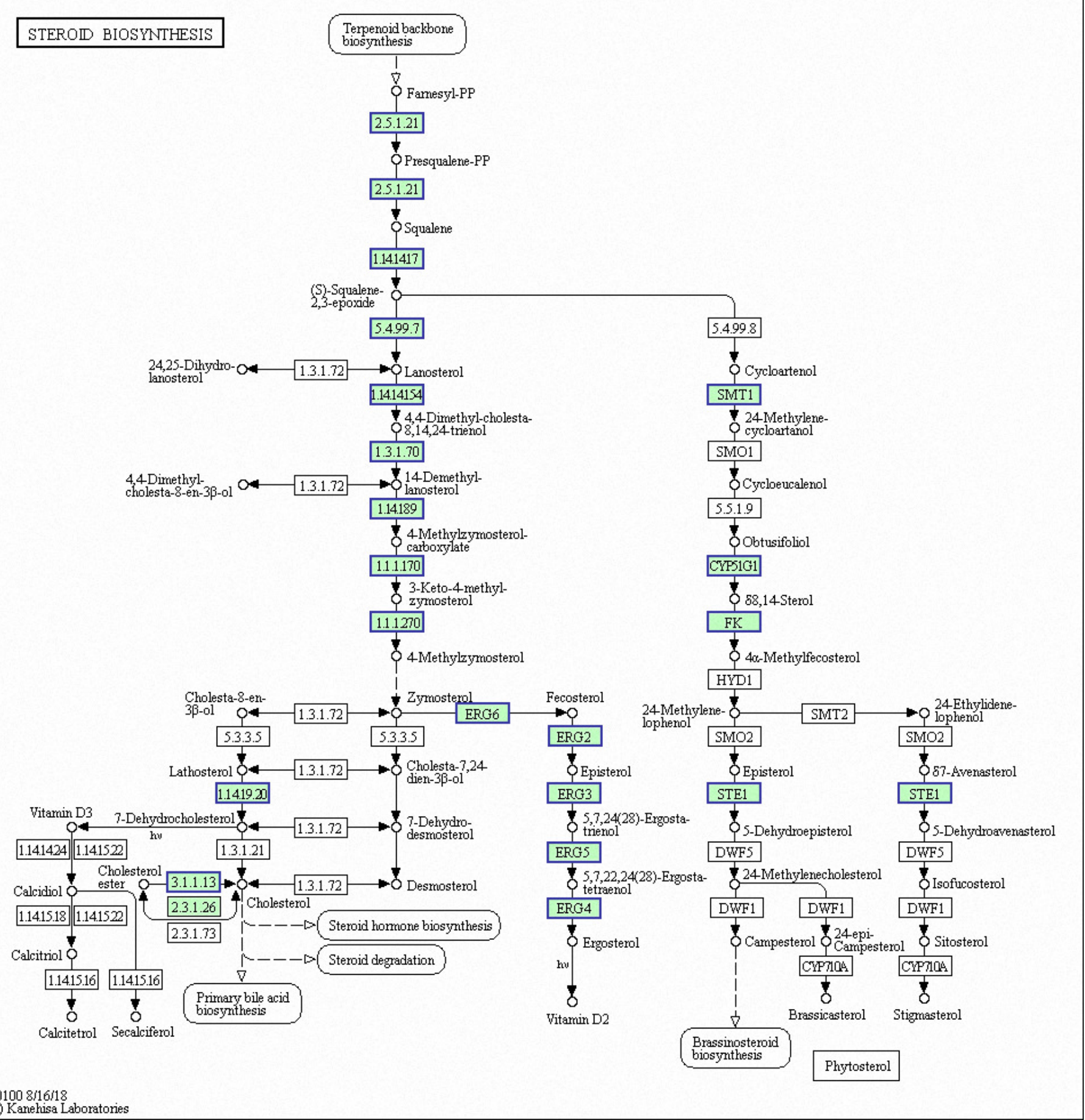


Figure 6

Significantly enriched KEGG pathways in fatty acid degradation.

DEGs with increased expression are marked in purple. Permission for publication was granted

by the Kyoto Encyclopedia of Genes and Genomes, Kyoto University, Japan.

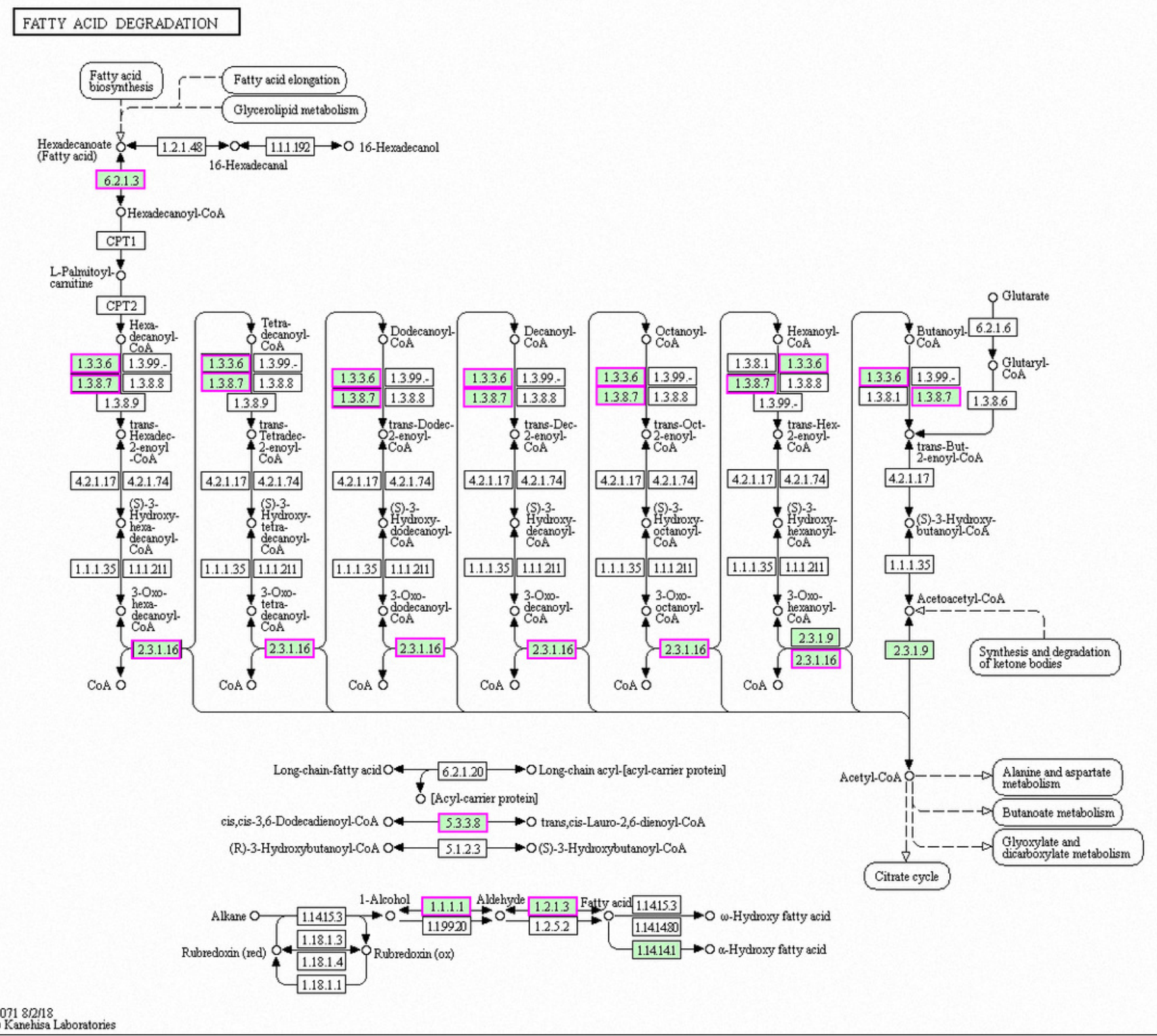


Figure 7

Significantly enriched KEGG pathways in oxidative phosphorylation.

DEGs with increased expression are marked in purple. Permission for publication was granted

by the Kyoto Encyclopedia of Genes and Genomes, Kyoto University, Japan.

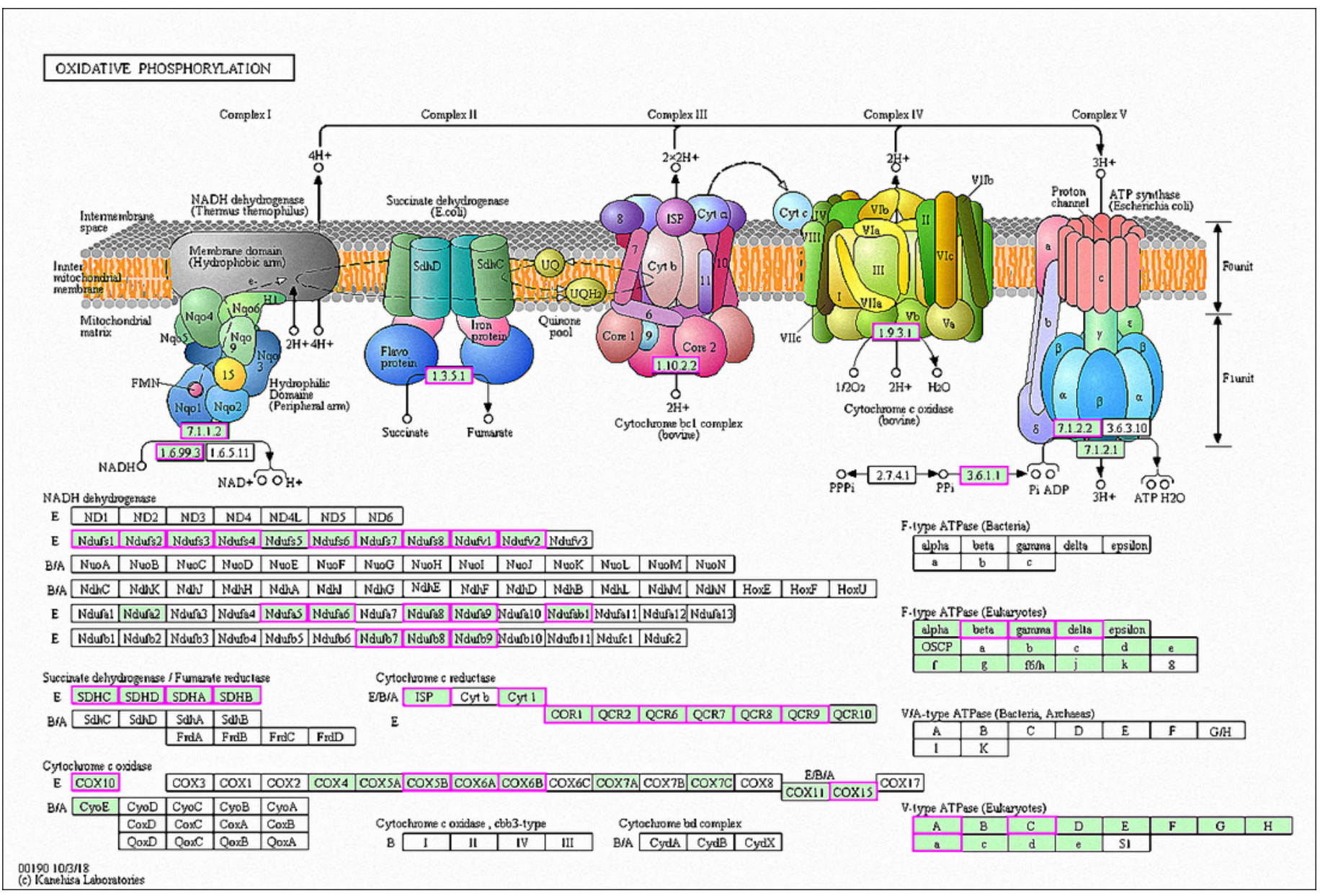




\section{Table $\mathbf{1}$ (on next page)}

Significantly enriched KEGG pathways for genes with increased expression after $6 \mathrm{~h}$ of MAF-1A treatment.

padj of $<0.05$ set as the significance threshold. 
1 Table 1. Significantly enriched KEGG pathways for genes with increased expression after $6 \mathrm{~h}$ of 2 MAF-1A treatment. padj of $<0.05$ set as the significance threshold.

\begin{tabular}{llll}
\hline KEGGID & Description & pvalue & padj \\
\hline cdu00190 & Oxidative phosphorylation & $3.34 \times 10^{-12}$ & $2.84 \times 10^{-10}$ \\
cdu04146 & Peroxisome & $2.02 \times 10^{-8}$ & $8.56 \times 10^{-7}$ \\
cdu00020 & Citrate cycle (TCA cycle) & $2.21 \times 10^{-5}$ & $6.27 \times 10^{-4}$ \\
cdu01200 & Carbon metabolism & $3.50 \times 10^{-5}$ & $7.44 \times 10^{-4}$ \\
cdu04111 & Cell cycle - yeast & $9.20 \times 10^{-5}$ & $1.56 \times 10^{-3}$ \\
cdu04011 & MAPK signaling pathway - yeast & $8.17 \times 10^{-4}$ & $1.16 \times 10^{-2}$ \\
cdu04113 & Meiosis - yeast & $2.35 \times 10^{-3}$ & $2.80 \times 10^{-2}$ \\
cdu00071 & Fatty acid degradation & $2.63 \times 10^{-3}$ & $2.80 \times 10^{-2}$ \\
cdu04136 & Autophagy - other & $4.03 \times 10^{-3}$ & $3.80 \times 10^{-2}$ \\
\hline
\end{tabular}

3 


\section{Table 2 (on next page)}

Significantly enriched KEGG pathways for genes with decreased expression after $6 \mathrm{~h}$ of MAF-1A treatment.

padj of $<0.05$ set as the significance threshold. 
1 Table 2. Significantly enriched KEGG pathways for genes with decreased expression after $6 \mathrm{~h}$ of MAF-1A treatment. padj of $<0.05$ set as the significance threshold.

\begin{tabular}{llll}
\hline KEGGID & Description & pvalue & padj \\
\hline cdu00100 & Steroid biosynthesis & $1.64 \times 10^{-8}$ & $1.40 \times 10^{-6}$ \\
cdu01230 & Biosynthesis of amino acids & $5.95 \times 10^{-7}$ & $2.53 \times 10^{-5}$ \\
cdu00270 & Cysteine and methionine metabolism & $4.80 \times 10^{-6}$ & $1.36 \times 10^{-4}$ \\
cdu01130 & Biosynthesis of antibiotics & $1.07 \times 10^{-5}$ & $2.27 \times 10^{-4}$ \\
cdu03010 & Ribosome & $3.01 \times 10^{-5}$ & $5.12 \times 10^{-4}$ \\
cdu03020 & RNA polymerase & $2.14 \times 10^{-4}$ & $3.03 \times 10^{-3}$ \\
cdu01110 & Biosynthesis of secondary metabolites & $3.68 \times 10^{-4}$ & $4.47 \times 10^{-3}$ \\
cdu03013 & RNA transport & $9.34 \times 10^{-4}$ & $8.83 \times 10^{-3}$ \\
cdu03008 & Ribosome biogenesis in eukaryotes & $9.35 \times 10^{-4}$ & $8.83 \times 10^{-3}$ \\
cdu00300 & Lysine biosynthesis & $2.31 \times 10^{-3}$ & $1.96 \times 10^{-2}$ \\
cdu01210 & 2-Oxocarboxylic acid metabolism & $2.66 \times 10^{-3}$ & $2.05 \times 10^{-2}$ \\
cdu00240 & Pyrimidine metabolism & $3.54 \times 10^{-3}$ & $2.40 \times 10^{-2}$ \\
cdu00260 & Glycine, serine and threonine metabolism & $3.67 \times 10^{-3}$ & $2.40 \times 10^{-2}$ \\
cdu00230 & Purine metabolism & $5.08 \times 10^{-3}$ & $3.09 \times 10^{-2}$ \\
\hline
\end{tabular}

3

4 


\section{Table 3 (on next page)}

Significantly enriched KEGG pathways for genes with increased expression after $18 \mathrm{~h}$ of MAF-1A treatment.

padj of $<0.05$ were set as the significance threshold. 
1 Table 3. Significantly enriched KEGG pathways for genes with increased expression after $18 \mathrm{~h}$ 2 of MAF-1A treatment. padj of $<0.05$ were set as the significance threshold.

\begin{tabular}{lccc}
\hline KEGGID & Description & p-value & padj \\
\hline cdu00220 & Arginine biosynthesis & $3.01 \times 10^{-4}$ & $7.21 \times 10^{-3}$ \\
cdu00250 & Alanine, aspartate and glutamate metabolism & $1.48 \times 10^{-3}$ & $1.77 \times 10^{-2}$ \\
\hline
\end{tabular}

3 


\section{Table 4 (on next page)}

Significantly enriched KEGG pathways for genes with decreased expression after $18 \mathrm{~h}$ of MAF-1A treatment.

padj of $<0.05$ set as the significance threshold. 
1 Table 4. Significantly enriched KEGG pathways for genes with decreased expression after $18 \mathrm{~h}$ of MAF-1A treatment. padj of $<0.05$ set as the significance threshold.

\begin{tabular}{|c|c|c|c|}
\hline KEGGID & Description & p-value & padj \\
\hline cdu01200 & Carbon metabolism & $1.65 \times 10^{-8}$ & $7.61 \times 10^{-7}$ \\
\hline $\mathrm{cdu} 01130$ & Biosynthesis of antibiotics & $6.21 \times 10^{-7}$ & $1.43 \times 10^{-5}$ \\
\hline cdu00190 & Oxidative phosphorylation & $5.58 \times 10^{-6}$ & $6.91 \times 10^{-5}$ \\
\hline cdu01110 & Biosynthesis of secondary metabolites & $6.01 \times 10^{-6}$ & $6.91 \times 10^{-5}$ \\
\hline $\mathrm{cdu} 00010$ & Glycolysis / Gluconeogenesis & $1.71 \times 10^{-5}$ & $1.57 \times 10^{-4}$ \\
\hline cdu00260 & Glycine, serine and threonine metabolism & $5.83 \times 10^{-4}$ & $4.47 \times 10^{-3}$ \\
\hline cdu01230 & Biosynthesis of amino acids & $1.77 \times 10^{-3}$ & $1.16 \times 10^{-2}$ \\
\hline cdu00680 & Methane metabolism & $3.62 \times 10^{-3}$ & $2.05 \times 10^{-2}$ \\
\hline cdu00520 & Amino sugar and nucleotide sugar metabolism & $4.02 \times 10^{-3}$ & $2.05 \times 10^{-2}$ \\
\hline cdu00052 & Galactose metabolism & $6.24 \times 10^{-3}$ & $2.87 \times 10^{-2}$ \\
\hline cdu00730 & Thiamine metabolism & $7.93 \times 10^{-3}$ & $3.20 \times 10^{-2}$ \\
\hline cdu00630 & Glyoxylate and dicarboxylate metabolism & $8.34 \times 10^{-3}$ & $3.20 \times 10^{-2}$ \\
\hline cdu00330 & Arginine and proline metabolism & $9.61 \times 10^{-3}$ & $3.24 \times 10^{-2}$ \\
\hline cdu00670 & One carbon pool by folate & $9.85 \times 10^{-3}$ & $3.24 \times 10^{-2}$ \\
\hline
\end{tabular}




\section{Table 5 (on next page)}

Significantly enriched KEGG pathways for genes in RG1, RG2 and UG1.

padj of $<0.05$ set as the significance threshold. 
1 2

\begin{tabular}{|c|c|c|c|c|}
\hline sort & KEGG ID & Description & p-value & padj \\
\hline RG1 & cdu00190 & Oxidative phosphorylation & $1.34 \times 10^{-5}$ & $2.27 \times 10^{-4}$ \\
\hline \multirow[t]{4}{*}{ RG2 } & cdu00220 & Arginine biosynthesis & $1.97 \times 10^{-5}$ & $2.56 \times 10^{-4}$ \\
\hline & cdu01130 & Biosynthesis of antibiotics & $4.09 \times 10^{-3}$ & $2.39 \times 10^{-2}$ \\
\hline & cdu01230 & Biosynthesis of amino acids & $5.52 \times 10^{-3}$ & $2.39 \times 10^{-2}$ \\
\hline & cdu01110 & Biosynthesis of secondary metabolites & $1.21 \times 10^{-2}$ & $3.92 \times 10^{-2}$ \\
\hline \multirow[t]{4}{*}{ UG1 } & cdu00650 & Butanoate metabolism & $1.82 \times 10^{-2}$ & $4.91 \times 10^{-2}$ \\
\hline & cdu00640 & Propanoate metabolism & $2.48 \times 10^{-2}$ & $4.91 \times 10^{-2}$ \\
\hline & cdu00410 & beta-Alanine metabolism & $2.64 \times 10^{-2}$ & $4.91 \times 10^{-2}$ \\
\hline & cdu00280 & Valine, leucine and isoleucine degradation & $2.81 \times 10^{-2}$ & $4.91 \times 10^{-2}$ \\
\hline
\end{tabular}

Table 5. Significantly enriched KEGG pathways for genes in RG1, RG2 and UG1. padj of < 0.05 set as the significance threshold. 


\section{Table 6(on next page)}

Significant enriched GO terms of RG1, RG2 and UG2.

padj of $<0.05$ set as the significance threshold for enrichment. 
1 Table 6. Significant enriched GO terms of RG1, RG2 and UG2. padj of $<0.05$ set as 2 the significance threshold for enrichment.

\begin{tabular}{|c|c|c|c|c|c|}
\hline sort & Category & GO ID & Description & p-value & padj \\
\hline \multirow[t]{2}{*}{ RG1 } & $\mathrm{BP}$ & GO:0006091 & $\begin{array}{l}\text { generation of precursor } \\
\text { metabolites and energy }\end{array}$ & $8.71 \times 10^{-5}$ & $7.14 \times 10^{-3}$ \\
\hline & $\mathrm{BP}$ & GO:0055114 & oxidation-reduction process & $8.49 \times 10^{-4}$ & $3.48 \times 10^{-2}$ \\
\hline \multirow[t]{6}{*}{ RG2 } & $\mathrm{BP}$ & GO:0016053 & organic acid biosynthetic process & $5.27 \times 10^{-4}$ & $2.11 \times 10^{-2}$ \\
\hline & $\mathrm{BP}$ & GO:0046394 & $\begin{array}{c}\text { carboxylic acid biosynthetic } \\
\text { process }\end{array}$ & $5.27 \times 10^{-4}$ & $2.11 \times 10^{-2}$ \\
\hline & $\mathrm{BP}$ & GO:0044283 & $\begin{array}{l}\text { small molecule biosynthetic } \\
\text { process }\end{array}$ & $1.56 \times 10^{-3}$ & $3.12 \times 10^{-2}$ \\
\hline & $\mathrm{BP}$ & GO:0006082 & organic acid metabolic process & $2.34 \times 10^{-3}$ & $3.12 \times 10^{-2}$ \\
\hline & $\mathrm{BP}$ & GO:0019752 & $\begin{array}{c}\text { carboxylic acid metabolic } \\
\text { process }\end{array}$ & $2.34 \times 10^{-3}$ & $3.12 \times 10^{-2}$ \\
\hline & $\mathrm{BP}$ & GO:0043436 & oxoacid metabolic process & $2.34 \times 10^{-3}$ & $3.12 \times 10^{-2}$ \\
\hline $\mathrm{UG} 2$ & $\mathrm{BP}$ & GO:0055114 & oxidation-reduction process & $3.53 \times 10^{-4}$ & $2.15 \times 10^{-2}$ \\
\hline
\end{tabular}

